\title{
Prognostic value of VEGFR2 immunoexpression in glioblastoma
}

\author{
Gemma Issus ${ }^{1,2}$, Sergi Mojal ${ }^{3}$, Joan Gibert ${ }^{4}$, Pilar Navarro ${ }^{4}$, Montserrat Arumi-Uria ${ }^{1}$, Dolores Naranjo-Hans ${ }^{1}$, Beatriz \\ Bellosillo ${ }^{1}$, Maria Martínez-Garcia ${ }^{5}$, Francesc Alameda*1,2 \\ ${ }^{1}$ Department of Pathology, Hospital del Mar, Barcelona, Spain \\ ${ }^{2}$ Universtat Autònoma de Barcelona, Barcelona, Spain \\ ${ }^{3}$ Biomedical Research Methods Consultant, IMIM (Hospital del Mar Medical Research Institute), Barcelona, Spain \\ ${ }^{4}$ Cancer Research Programme, IMIM (Hospital del Mar Medical Research Institute), Barcelona, Spain \\ ${ }^{5}$ Department of Oncology, Hospital del Mar, Barcelona, Spain
}

Received: January 22, 2019

DOI: $10.5430 /$ jst.v10n1p1
Accepted: November 18, $2019 \quad$ Online Published: October 10, 2019

URL: https://doi.org/10.5430/jst.v10n1p1

\begin{abstract}
Glioblastoma is the most frequent and aggressive primary tumor of the central nervous system. Prognosis is poor, with a median survival of 15 months after diagnosis. Various tumor biomarkers show prognostic value for glioblastomas, including VEGFR2, which is a receptor of VEGF related to the growth of the blood vessel network. VEGFR2 expression associates with poor prognosis in some tumors. Here we studied the prognostic value of the VEGFR2 immunohistochemical expression in glioblastoma. We used tissue microarrays to analyze 45 surgically excised samples from glioblastomas. Clinical data (age, sex, and Karnofsky Performance Status [KPS]) and morphological data (tumor necrosis, palisading, and vascular thrombosis) were collected. We performed a molecular study of MGMT and IDH1 expression (which are potential prognostic factors for glioblastomas) and an immunohistochemical study of VEGFR2 expression. Our results indicate that age, KPS, tumor necrosis, vascular thrombosis, treatment (STUPP versus other), and VEGFR2 immunoreactivity were related to prognosis $(p<.005)$. In a multivariate analysis, only age $>65$ years (Hazard Ratio (HR) (95\% CI): $4.9(2.1-11.4), p<.01)$, and VEGFR2 immunoexpression (HR (95\% CI): 2.8 $(1.3-6.1), p=.008)$, were found to have a statistically significant relation to prognosis. We conclude that immunohistochemical evaluation of VEGFR2 provides added prognostic value to the study of glioblastoma.
\end{abstract}

Key Words: Glioblastoma, VEGFR2, IDH1, MGMT, Prognosis

\section{INTRODUCTION}

Glioblastoma (GB) is the most frequent and aggressive primary tumor of the central nervous system (CNS). GB comprise $12 \%-15 \%$ of all intracranial neoplasms, and $50 \%-60 \%$ of all astrocytic tumors, and is more frequent in adults aged between 45 and 70 years. ${ }^{[1]}$ These tumors are formed by poorly differentiated pleomorphic astrocytic cells, with marked atypia, mitosis, palisading, necrosis, vascular proliferation, and endothelial hyperplasia (1). GB can appear de novo (primary) or from diffuse (grade II) or anaplastic (grade III) astrocytoma (secondary). ${ }^{[1]}$ Some tumor markers have been identified as potential prognostic factors, such as isocitrate-dehydrogenase (IDH) mutation. ${ }^{[1]}$ The most frequent mutation is the IDH1 R132H mutation. ${ }^{[2]}$ The prognostic value of IDH1 mutation has been reflected in a new classification issue by the World Health Organization. ${ }^{[1]}$ VEGF is related to poor prognosis and tumor progression ${ }^{[3]}$ VEGF is highly expressed in palisading tumor cells. ${ }^{[4]}$ These

\footnotetext{
*Correspondence: Francesc Alamed; Email: falameda@hospitaldelmar.cat; Address: Department of Pathology, Hospital del Mar Passeig Marítim 25-29, 08003 Barcelona, Spain. 
cells are subjected to high degrees of hypoxia and express hypoxia-inducible factor 1 (HIF1) and proangiogenic factors, such as VEGF and interleukin 8 (IL-8), and they are strongly associated with microvascular hyperplasia. ${ }^{[4,5]}$ The association between an increase of gene expression related with hypoxia and neovascularization has been published. ${ }^{[6]}$ This increased expression is more intense for wild-type IDH1 than for mutated IDH1. ${ }^{[6]}$ GBs containing mutated IDH1 are less angiogenic, have smaller blood vessels, and are associated with less hypoxia than those containing wild-type IDH1. ${ }^{[7]}$ Increased hypoxia is associated to an increase in invasiveness, stem cell maintenance, treatment resistance, and angiogenesis. ${ }^{[8]}$ Hypoxia triggers VEGF expression, and this is a critical step for abnormal vascular expression ${ }^{[9]} \mathrm{VEGF}$ has two receptors, namely VEGFR1 and VEGFR2, which are both expressed on the surface of GB cells. However, VEGFR2 appears to mediate many interactions produced by VEGF. ${ }^{[10]}$ VEGFR2 is stimulated under normal concentrations of VEGF, while VEGFR1 is maintained inactive. ${ }^{[10,11]}$ VEGFR1 is activated in response to high VEGF levels and acts as an inhibitor of VEGFR2. ${ }^{[10]}$ VEGFR2 expression is regulated by MMP2 ${ }^{[3]}$, TGF-beta, ${ }^{[12]}$ and HIF1. ${ }^{[1]}$ The co-expression of VEGF and its receptors is regulated in an autocrine manner. ${ }^{[1]}$ Importantly, VEGFR2 is expressed by tumor cells. Glioma stem-like cells participate in tumor neovascularization by transdifferentiation into vascular endothelial cells. These cells show preferential expression for VEGFR2, and knockdown of VEGFR2 in these cells

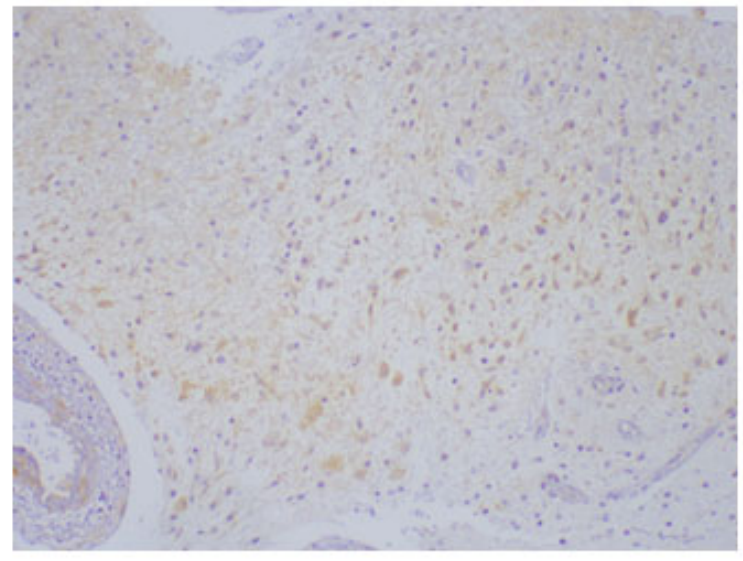

A reduces their self-renewal capacity and promotes vascularization. VEGFR2 is an essential molecule for sustaining the stemness of glioma stem-like cells as well as for their capacity to initiate tumor vasculature, and thus for the development of the tumor. ${ }^{[13]}$ Treatment of GB is based on surgery and radio- and chemotherapy ${ }^{[14]}$ but prognosis is poor. The mean survival following diagnosis is 15 months. Long-term survivors are understood as those patients still alive at three years after diagnosis. ${ }^{[4]}$ Various parameters are associated with prognosis, such as age, the Karnofsky performance status (KPS) at diagnosis, and IDH1 mutation status. ${ }^{[1]}$ Furthermore, some studies have emphasized the prognostic value of several histological features, such as tumor necrosis and giant multinucleated tumor cells. ${ }^{[15]}$ AntiVEGF treatment induces a hypoxic tumor microenvironment, thereby favoring a metabolic change in the tumor cells that leads to enhanced tumor cell invasion. ${ }^{[16] ~ V E G F ~ b l o c k a d e ~}$ restores and increases MET activity in GB, while inducing a program reminiscent of the epithelial-to-mesenchymal transition, highlighted by a T-cadherin to N-cadherin switch and enhanced mesenchymal features. Inhibition of MET in mouse models blocks the VEGF-induced mesenchymal transition and invasion, resulting in a substantial increase in mouse survival, with interesting clinical potential. ${ }^{[17]}$ Here we studied the prognostic value of various clinical, morphological, and molecular data, with particular emphasis on VEGFR2 immunoexpression (VEGFR2-IE).

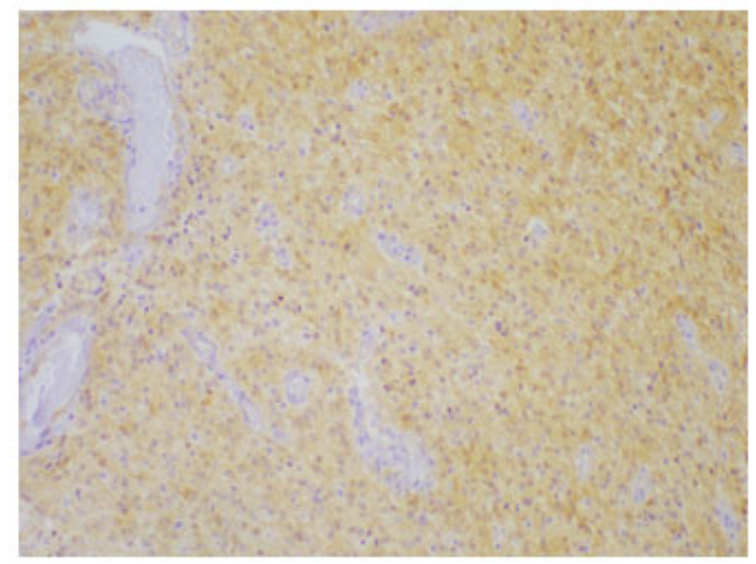

B

Figure 1. IHC analysis in the glioblastoma tissue microarray. VEGFR2-IE. Different levels of expression (low or high) are shown. VEGFR2-IE $<25 \%$ (A) $>25 \%$ (B). Magnification: $10 \times$.

\section{MethodS}

\subsection{Patients}

Fifty-three consecutive patients diagnosed with GB between 2008 and 2015 were reviewed. Inclusion criteria were to have all pathological information required for the study, including follow-up data. Eight patients were excluded due to lack of follow-up data. Forty-five cases were included in the study. Sex, age, KPS scale, tumor location, surgical 
resection, and length of survival were taken from clinical records. Survival was taken as the number of months patients survived from the day after surgery. Furthermore, the histopathological slides of these patients were reviewed, and the following parameters were examined: tumor necrosis, endothelial hyperplasia, palisading necrosis, and vascular thrombosis.

\subsection{Molecular methods}

The methylation status of the MGMT promoter, and the mutational status of IDH1, were studied in all of these cases. For this, DNA was extracted from two $15-\mu \mathrm{m}$ sections of paraffin-embedded tissue using the QIAamp DNA Mini Kit (QIAGEN GmbH, Hilden, Germany), following the manufacturer's protocol. For tissue with less than $50 \%$ of tumoral cells, the tumor tissue was macrodissected manually. For analysis of MGMT promoter methylation, $500 \mathrm{ng}$ of extracted DNA was analyzed by bisulfite treatment using the EZ DNA Methylation-Gold Kit (Zymo Research Corporation, Irvine, CA, USA). DNA methylation patterns in the $\mathrm{CpG}$ island of the MGMT gene were determined by methylation-specific PCR (MSP) using primers specific for either methylated or modified non-methylated DNA, as previously described. ${ }^{[18]}$ The IDH1 R132H mutation was assessed by an allelic discrimination assay, which detects low levels of mutant sequence, using TaqMan probes specific for the wild-type (FAM-5'-CATAGGTCGTCATGCTTA-3') and R132H mutant (VIC-5' - CATAGGTCATCATGCTTA-3') alleles. PCR amplification and detection were performed on an ABI Prism 7500FAST Genetic Analyzer (Applied Biosystems) with an initial step of $10 \mathrm{~min}$ at $95^{\circ} \mathrm{C}$, followed by 40 cycles of $15 \mathrm{sec}$ at $95^{\circ} \mathrm{C}$ and $1 \mathrm{~min}$ at $60^{\circ} \mathrm{C}$.

\subsection{TMA}

A tissue microarray (TMA) was built using a 1-mm needle in a microarrayer (Chemicon, California, USA). Three TMAs were obtained, each containing three or four representative areas of each tumor.

\subsection{Immunohistochemical methods}

Slides of 3-micron-thick tissue samples were obtained from each TMA and analyzed by immunohistochemical staining for VEGFR2. For this, slides were deparaffinized, and antigen was retrieved for $10 \mathrm{~min}$ with citrate buffer $0.01 \mathrm{M}$ $(\mathrm{pH} 6.0)$ at $120^{\circ} \mathrm{C}$. Sections were treated with $3 \% \mathrm{H} 2 \mathrm{O} 2$ to quench endogenous peroxidase activity and blocked with PBS 1\% FBS. Primary antibody (VEGFR2 from Cell Signaling, rabbit monoclonal antibody D5B1 1/500) was added at the corresponding dilution overnight at $4^{\circ} \mathrm{C}$. Pre-immune rabbit or mouse IgG was used as a negative control. As the secondary antibody, HRP-conjugated (Envision+, Dako) anti-rabbit Ig was used. After washes with PBS, DAB (3, 3-diaminobenzidine) was added as a chromogenic substrate (Dako). Sections were counterstained with hematoxylin \& eosin, and then dehydrated and mounted. Immunohistochemistry was examined in an Olympus BX61 microscope, and the cellSens software was used for image acquisition. TMAs were examined by two pathologists (GI and FA). The method of evaluation was to observe the positivity of tumor cells in the cytoplasm (see Figure 1 A and B). The percent of positive cells was calculated. An agreement was reached between the two pathologists when the difference in evaluation of each case was $<10 \%$; if the difference was $\geq 10 \%$, the sample was re-evaluated using a multiheaded microscope. The median expression of VEGFR-IE was calculated. Of the different cut-offs tested for VEGFR2-IE, $\leq 25 \%$ was considered to be the most discriminant point with respect to patient survival time. VEGFR2-IE was considered positive when at least $25 \%$ of the tumor cells showed VEGFR2 immunostaining. ${ }^{[14]}$ (see Figure 2)

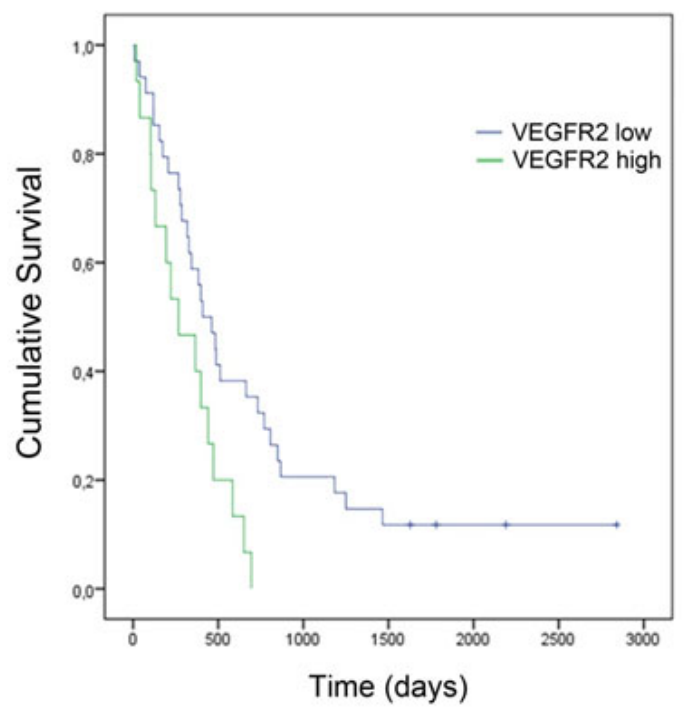

Figure 2. VEGFR2-IE and Kaplan-Meier survival analysis. Patients showing high VEGFR2-IE ( $>25 \%$ of tumor cells positive for VEGFR2) (green) display lower survival than those with low VEGFR2-IE $(<25 \%$ of tumor cells positive for VEGFR2) (blue)

\subsection{Ethical Considerations}

This work was approved by the Ethical Committee of the Hospital del Mar with number 2013/5122

\subsection{Statistical analysis}

Mean and standard deviations were used to describe age, while frequencies and percentages were used for categorical variables. Survival time in the different analysis was expressed as median and 95\% confidence interval (CI). Kaplan 
Meier curves and the log rank test were used to compare the survival times between different variables. To determine the factors related to prognosis, multivariate Cox regression analyses were performed for cases with wild-type IDH1. In this case, hazard ratios with their $95 \%$ CI were shown. All analyses were performed with SPSS 21.0 (IBM Corp.).

\section{Results}

A total of 45 patients with GB were included in the study. The clinical information for these patients is summarized in Table 1.

Table 1. Clinical and morphological data of GB patients. Bivariate analysis, based on all the patients

\begin{tabular}{llll}
\hline Data & (n) & Median $($ CI95\%) & $\boldsymbol{P}^{*}$ \\
\hline Sex & Wo (16) & $88(64-112)$ & .062 \\
Age & Ma (29) & $145(103-187)$ & \\
& $<=65(29)$ & $155(54-256)$ & .000 \\
Karnofsky & $>65(16)$ & $57(33-82)$ & \\
& $<=70(22)$ & $88(64-112)$ & .027 \\
Resection & $>70(23)$ & $160(87-233)$ & \\
& Pa (15) & $88(18-158)$ & .848 \\
Necrosis & To (30) & $131(81-181)$ & \\
& No (4) & $126(0-875)$ & .038 \\
Endot. hyper. & Yes (41) & $113(74-151)$ & \\
& No (9) & $159(0-497)$ & .102 \\
Palisading & No (21) & $113(81-144)$ & \\
& Yes (24) & $108(56-159)$ & .663 \\
Thrombosis & No (10) & $240(187-168)$ & \\
& Yes (35) & $105(76-134)$ & .040 \\
Treatment $(*)$ & STUPP (23) & $155(116-193)$ & .018 \\
& Other (19) & $63(29-98)$ & \\
\hline
\end{tabular}

Note. Median: Median survival (in months) *: Log-Rank test. Sex, Ma= Male; Wo= Female; Resection, $\mathrm{To}=$ Total or subtotal; $\mathrm{Pa}=$ Partial or just biopsy; Endot. hyper. $=$ Endothelial hyperplasia; Multi. Cells= Multinucleated giant cells. Treatment $(*): 42$ patients. 3 patients did not receive treatment after surgery.

\subsection{Clinical and surgical data}

The median survival time of patients was 12 months (95\% CI: 9.15-14.8). Two patients (4.4\%) died before 1.3 months, 10 (22.2\%) between 1.3 and 5.7 months, $11(24.4 \%)$ between 6.3 and 12 months, 12 (26.7\%) between 12.6 and 22.8 months, 5 (11.1\%) between 24.1 and 27.9 months, and 5 (11.1\%) after 28 months. with a maximum survival time of 72 months. The study included 29 men (64.4\%) and 16 women $(35.6 \%)$. Marginally significant differences were observed for patient survival and sex $(p=.062$; see Table 1$)$. The mean (SD) age of all patients was 58.5 (14.1) years (range 29-81 years). The distribution of patient age was as follows: 5 patients were between 29 and 39 years, 9 between 40 and 49 years, 8 between 50 and 59 years, 11 between 60 and 69 years, and 4
12 were 70 years or older. In total, 29 patients were 65 years old or younger, and 16 patients were over 65 years old. Age was split at 65 years as standard treatment is recommended for patients under this age, and treatments are not well established for those over 65 . The survival time was 15.5 months (95\% CI: 5.4-25.6) for patients aged 65 years or younger, and 5.7 months (95\% CI: 3.3-8.2) for those over 65 years. The difference in survival time was statistically significant ( $p$ $<.001$; see Table 1 ). In the 30 cases involving the temporal lobe, tumors were either limited to only the temporal lobe (18 cases) or also extended to the parietal lobe (five cases), the frontal lobe (four cases), both the frontal and parietal lobes (two cases) or the occipital lobe (one case). In the 13 cases involving the frontal lobe, most tumors were limited to only the frontal lobe (12 cases), with one case in which the tumor also extended to the parietal lobe. The tumor was located exclusively in the parietal lobe in one patient, and exclusively in the occipital lobe in one patient. None of the patients in this group had tumors located in central structures. The KPS scale at diagnosis was under 70 for 22 cases, and over 70 for 23 cases. Median survival of patients for KPS under 70 was 8.8 months (95\% CI: 6.4-11.2), and for over 70, 16.0 months (95\% CI: 8.7-23.3). The difference was statistically significant ( $p=.027$; see Table 1). Surgical resection was total in 16 cases $(35.6 \%)$, subtotal (i.e., more than $80 \%$ of the tumor) in 14 cases $(31.1 \%$ ), and partial (i.e., less than $80 \%$ of the tumor) in 12 cases $(26.6 \%)$. Three cases were biopsied only $(6.7 \%)$. The evaluation of extension of surgical resection was done with postoperative radiological evaluation. Two patient groups were established: i) total or subtotal surgical resections (30 cases), and ii) partial resection or biopsy (15 cases). The differences in survival between these two groups was not statistically significant ( $p=.848$; see Table 1$)$.

\subsection{Treatment}

Three patients did not receive post-surgical treatment. In patients who received such treatment (42), 23 were treated with Stupp's protocol (e.g., postoperative radiotherapy, of $60 \mathrm{~Gy}$, with concomitant and adjuvant temozolamide for 6 cycles)(19), and 19, with another treatment (UCP 10, TMZ 1, BCN1, 1, QT-RT 1, RT 6). The patients treated with Stupp's protocol survived a median of 15.5 months $(95 \%$ CI: 11.6-19.3) while those who received other treatments survived a median of 6.3 months (95\% CI: 2.9-9.8). The difference was statistically significant ( $p=.018$; see Table 1).

\subsection{Histopathological data}

Results of the histopathological analyses of the 45 cases revealed: tumor necrosis in 41 cases $(91.1 \%)$, endothelial hyperplasia in 36 cases $(80 \%)$, palisading necrosis in 24 
cases $(53.3 \%)$, and vascular thrombosis in 35 cases $(77.8 \%)$ (see Table 2). Patients with vascular thrombosis survived a median of 10.5 months (95\% CI: 7.6-13. 4), while patients without vascular thrombosis survived a median of 24.0 months (95\% CI: 18.7-29.4) Vascular thrombosis was associated with poor prognosis $(p=.040)$ (see Table 1).

Table 2. Molecular and immunohistochemical data of GB patients. Bivariate analysis, based on all the patients

\begin{tabular}{llll}
\hline Gene/protein & \multicolumn{1}{c}{$\mathbf{n}$} & Median $(\mathbf{C I 9 5 \% )}$ & $\boldsymbol{P}^{*}$ \\
\hline MGMT & MET (14) & $119(71-168)$ & 0099 \\
& UNMET ( 31) & $113(69-157)$ & \\
IDH1 & MUT (8) & $94(0-269)$ & 0075 \\
& NO MUT (37) & $12(94-146)$ & \\
VEGFR2 & $<=25(25)$ & $131(25-237)$ & 0029 \\
& $>25(20)$ & $108(90-125)$ & \\
VEGFR2 & $<=25(17)$ & $131(0-268)$ & 0069 \\
(IDH1WT) & & & \\
\hline
\end{tabular}

Note. Median: Median survival (in months) *:Log-Rank test.

\subsection{Molecular and immunohistochemical data}

The MGMT promoter was found to be methylated (MGMT$\mathrm{PM})$ in 14 cases $(30.4 \%)$, and the IDH1R132H mutation was present in 8 cases (17.7\%). All of them were related to survival in a marginally significant manner $(p=.099$ and $p=$ .075) (see Table 2).Five of the cases showing IDH1 wild type, have less than 50 years old. In all of them the surgical resection of the tumor was complete. No study of IDH2 mutation was performed. The IHD2 mutations are very infrequent in GB. ${ }^{[2]}$ The mean positive staining for VEGFR2-IE was $28.2 \%$ (range $0-100 \%$ ) of cells. Twenty-five cases $(55.6 \%)$ comprised $\leq 25 \%$ cells with VEGFR2-IE, and 20 cases $(44.4 \%),>25 \%$. Survival analysis showed a correlation between VEGFR2-IE $>25 \%$ and poor prognosis of the patient. Cases with VEGFR2 IE $>25 \%$ correlated with patient survival of a median of 10.8 months (95\% CI: 9.0-12.5), while those $\leq 25 \%$, with a survival of a median of 13.1 months (95\% CI: 2.5-23.7) ( $p=.029$; see Table 2) . We performed bivariate analysis of VEGFR2-IE in cases that had wild-type IDH1 (IDH1-WT) and found that VEGFR2-IE higher than $25 \%$ correlated with poor prognosis, with a marginal statistical significance ( $p=.069$; see Table 2$)$. Of the cases in which IDH1 was mutated cases, none had more than $25 \%$ of cells positive for VEGFR-IE. In summary, in univariate analysis, the parameters influencing the prognosis were age, KPS, vascular thrombosis, and VEGFR2 immunoexpression. Multivariate analysis using these parameters revealed that only age ( $>65$ years old; HR 4.90; 95\% CI: $2.10-11.4, p<$ $.001)$ and positive VEGFR2-IE (> 25\%; HR 2.82; $95 \%$ CI: $1.31-6.08, p=.008)$ were factors that correlated to prognosis, and in particular, with a better prognosis in patients under 65 years of age and low VEGFR2-IE (see Table 3).

Table 3. Multivariate Cox regression analysis of prognosis

\begin{tabular}{ccc}
\hline Variable & HR $(\mathbf{9 5 \%}$ CI $)$ & $\boldsymbol{P}$ value \\
\hline VEGFR2-IE $>25 \%$ & $282(131-608)$ & .008 \\
AGE $(>65)$ & $490(210-114)$ & $<.001$ \\
\hline
\end{tabular}

\section{Discussion}

Glioblastoma (GB) is the most frequent and aggressive primary tumor of the CNS. Importantly, angiogenesis, which supports GB development and progression, has been reported as a major reason for the poor prognosis of these tumors. ${ }^{[3]}$ The overexpression of VEGF is related to hypoxia, ${ }^{[4]} \mathrm{mi}^{-}$ crovascular hyperplasia, ${ }^{[4,5]}$ and poor prognosis. ${ }^{[3]}$ In fact, hypoxia triggers VEGF expression, and this is a clue for abnormal vascular expression in GBs. ${ }^{[9]}$ VEGFR2 appears to mediate many of the interactions of VEGF. ${ }^{[10]}$ The coexpression of VEGF and its receptors is regulated in an autocrine manner. ${ }^{[11]}$ VEGF expression is higher in tumor areas that are under hypoxia ${ }^{[11]}$ in which the hypoxia factor 1 (HIF1) is highly expressed. Hypoxia is related to neovascularization, ${ }^{[6]}$ and angiogenesis is determined by VEGFR2 expression. ${ }^{[12]}$ Thus, there is a relationship between the hypoxia and the expression of VEGF and its receptors. Here, we have demonstrated the prognostic value of immunoexpression of VEGFR2 (VEGFR2-IE) in GB when VEGFR2 is expressed $>25 \%$ of tumor cells. Specifically, tumors showing $>25 \%$ VEGFR2-IE correlated with a lower survival time of patients as compared to those that had $\leq 25 \%(10.8$ months as compared to 13.1 months; $p=.029)$. The levels of VEGFR2-IE were higher in hypoxic areas, which are characterized by vascular proliferation, necrosis, and thrombosis. These observations may explain the relationship between poor survival, tumor necrosis, and thrombosis. Accordingly, we found that the mean percentage of cells with VEGFR2-IE was $31.4 \%$ in cases with tumor necrosis, as compared to $1.5 \%$ in cases without tumor necrosis. However, although necrosis in the present series was found to be related to poor prognosis, there were only four cases without necrosis: one after complete surgery, two after partial surgery, and one with only a biopsy. Thus, there are too few cases to consider this to have statistical value. Note that the relationship between poor prognosis and tumor necrosis has been documented in the literature; ${ }^{[10]}$ however, these data did not take into account expression of VEGFR2. We also observed potential relationships between VEGFR2-IE and thrombosis, endothelial hyperplasia, and palisading. Specifically, the mean levels of cells with VEGFR2-IE were: $33.5 \%$ in cases with thrombosis and $12.2 \%$ in cases without; $30.0 \%$ in cases with endothelial hyperplasia and $24.0 \%$ in cases without; and $42.9 \%$ in cases with palisading, and $12 . \%$ in cases without. 
A recent publication has demonstrated that wild-type IDH1 gliomas (grade II) and glioblastomas undergo a more intense hypoxia degree, with higher levels of expression of VEGF (which is mediated by some angiogenic factors expressed in endothelial cells) as well as of TGFbeta and HIF1. ${ }^{[6]}$ In accordance with these data, we have found an inverse correlation between the presence of VEGFR2-IE and mutated IDH1 with patient prognosis. The cases we studied with mutated IDH1 showed a mean VEGFR2-IE of $2.6 \%(0-8.25)$, while cases with wild-type IDH1 showed a mean VEGFR2-IE of $34.5 \%$ (0-100). These data highlight the prognostic value of VEGFR2-IE in GBs that contain wild-type IDH1. As a limitations of this work, we would like the point to the variety of treatments that patients underwent, which somewhat limits the results of this work. Furthermore, although we could have studied IDH mutations other than the more frequent IDH1 mutation, we considered it to be out of the scope of this work.

\section{Conclusion}

Our work indicates that measuring immunoexpression of VEGFR2 can provide a helpful biomarker for GB prognosis if introduced into the routine exams of GB, especially for those tumors that contain wild-type IDH1.

\section{FUNDING}

This work was supported by grants from the Fundació La Marató (num.20130332) to FA, and the Spanish Ministerio de Economía y Competitividad/ ISCIIIFEDER (PI14/00125; PI17/00199) and the "Generalitat de Catalunya" (2017/SGR/225) to PN.

\section{ACKNOWLEDGEMENTS}

We thank Marta Lorenzo, Ariadna Torner, and $\mathrm{M}^{a}$ Carmen Vela for technical support.

\section{CONFlicts OF InTEREST Disclosure}

The authors declare no conflict of interest

\section{REFERENCES}

[1] WHO classification of Tumors of the Central Nervous System. IARC. 4th ed revised. Lyon, 2016.

[2] Yan H, Parsons DW, Jin G, et al. IDH1 and IDH2 mutations in gliomas. N Eng J Med. 2009; 360 (8): 765-73. https : //doi . org/ 10.1016/S0513-5117(09) 79085-4

[3] Du R, Petritsch C, Lu K, et al. Metalloproteinase 2 regulates vascular patterning and growth affecting tumor cell survival and invasion in GBM. Neuro Oncol. 2008; 10(3): 254-64. PMid:18359864. https://doi.org/10.1215/15228517-2008-001

[4] Brat D. JGlioblastoma: biology, genetics, and behavior. American Society of Clinical Oncology Educational Book / ASCO. American Society of Clinical Oncology. Meeting, 102-7. 2012

[5] Zagzag D, Lukyanov Y, Lan L, et al. Hypoxia-inducible factor 1 and VEGF upregulate CXCR4 in glioblastoma: implications for angiogenesis and glioma cell invasion. Lab Invest. 2006; 86 (12): 1221-1232. PMid:17075581. https://doi.org/10.1038/labi nvest. 3700482

[6] Zhang L, He L, Lugano R, et al. IDH mutation status is associated with distinct vascular gene expression signature in low grade gliomas. Neuro Oncol. 2018; 20(11): 1505-16. PMid:29846705. https://doi.org/10.1093/neuonc/noy088

[7] Conroy S, Wagemakers $M$, Watenkamp AM, et al. Novel Insight into vascularization patterns and angiogenic facors in GB subclasses. J. Neurooncol. 2017; 131 (1): 11-20. PMid:27633774. https://doi.org/10.1007/s11060-016-2269-8

[8] Mahase S, Ratteni RN, Wesseling P, et al. Hypoxia mediated mechanisms associated with angiogénesis treatment resistance in glioblastomas. Am J Pathol. 2017; 187 (5): 940-953. PMid:28284719. https://doi.org/10.1016/j.ajpath.2017.01.010

[9] Paolicchi E, Gemigrani F, Krstic-Demonacos M, et al. Targeting hypoxic response for cancer therapy. Oncotarget 2016; 7(12): 13464 13478. PMid:26859576. https://doi .org/10.18632/oncotar get. 7229

[10] Xu C, Wu X, Zhu J. VEGF promotes proliferation of human glioblastoma multiforme stem-like cells through VEGF receptor 2. Scientific World Journal. 2013, 417413. PMid:23533349. https://doi.or $\mathrm{g} / 10.1155 / 2013 / 417413$
[11] Knizetova P, Ehrmann J, Hlobilkova A, et al. Autocrine regulation of gliobñastoma cell cycle progression, viability and radioresistance through the VEGF-VEGFR2 ( KDR ) interplay. Cell Cycle. 2008; 7(16), 2553-2561. PMid:18719373. https://doi.org/10.4161/ cc.7.16.6442

[12] Kuczynsky EA, Patten SG, Coomber BL. VEGFR2 expression and TGF-Beta signaling and recurrent high-grade human glioma. Oncology. 2011; 81(2): 126-34. PMid:21985798. https ://doi .org/10 $.1159 / 000332849$

[13] Yao X, Ping Y, Liu Y, et al. Vascular Endothelial Growth Factor Receptor 2 (VEGFR-2) Plays a Key Role in Vasculogenic Mimicry Formation, Neovascularization and Tumor Initiation by Glioma Stem-like Cells. PLoS ONE. 2013; 8(3): 112. https://doi.org/10.1371/annotation/aed5b555-b82 6-4591-8aa6-284ad888627d

[14] Fomby P, Cherlin AJ. NIH Public Access. 2011; 72(2): 181-204. PMid:21918579. https://doi.org/10.1177/00031224070720 0203

[15] Homma T, Fukushima T, Vaccarella S, et al. Correlation among pathology, genotype and patient outcome in glioblastomas. J Neuropathol Exp Neurol. 2006; 65(9): 846-54. PMid:16957578. https : //doi.org/10.1097/01.jnen.0000235118.75182.94

[16] Keunen O, Johansson M, Oudin A, et al. Anti-VEGF treatment reduces blood supply and increases tumor cell invasion in glioblastoma. Proc Nat Acad Sci USA. 2011; 108(9): 3749-3754. PMid:21321221. https://doi.org/10.1073/pnas. 1014480108

[17] Lu KV, Chang JP, Parachoniak CA, et al. VEGF inhibits tumor cell invasion and mesenchymal transition through a MET-VEGFR2 complex. Cancer Cell. 2012; 22(1): 21-35. PMid:22789536. https: //doi.org/10.1016/j.ccr.2012.05.037

[18] Esteller M, Hamilton SR, Burger PC, et al. Inactivation of DNA repair gene $=5$-methilguanine-DNA methyltransferase by promoter hypermethylation is a common event in primary human neoplasia. Cancer Res. 1999; 59(4): 793-7.

[19] Stupp R, Mason WP, van der Bent MJ, et al. Radiotherapy plus concomitant and adjuvant temozolamide for glioblastoma. N Eng J Med 2005; 352: 987-996. PMid:15758009. https://doi .org/10.105 6/NEJMoa043330 